\title{
3D Model of Historical Vltava River Valley: Combination of Sources
}

\author{
Jiri Cajthaml $^{\mathrm{a},}$, Darina Kratochvilova ${ }^{\mathrm{a}}$, Tomas Janata ${ }^{\mathrm{a}}$ \\ ${ }^{a}$ Czech Technical University in Prague, Department of Geomatics, Jiri Cajthaml, jiri.cajthaml@fsv.cvut.cz, Darina Kratochvilova, \\ darina.kratochvilova@fsv.cvut.cz,TomasJanata,tomas.janata@fsv.cvut.cz \\ * Corresponding author
}

\begin{abstract}
Within the framework of the project "Vltava - transformation of historical landscape as a result of floods, dams creation and land-use changes along with cultural and social activities in the river neighbourhood" funded by the Ministry of Culture of the Czech Republic, we deal with many aspects of the Vltava River, considering the significant changes that have taken place in its history. In this paper, we are focusing on the reconstruction of the historic Vltava valley as of 1950s, the period just before the construction of the dam reservoirs cascade on the Vltava River. The aim is to create DTM, which will be the basis of visualizations and analyses of the old river bed.
\end{abstract}

Keywords: 3D model, old maps, Vltava River, vectorization

\section{Introduction}

The Vltava River is the longest and apparently the most famous river in the Czech Republic. It has a very interesting history, because the river and its surroundings were greatly influenced by the construction of dams and water reservoirs in the $20^{\text {th }}$ century. We decided to focus on the area flooded with water of the reservoirs in our research. In these areas, both settlements and interesting natural monuments have disappeared. The aim of our work is to reconstruct the original valley of the Vltava River before construction of dams. See more about our project in (Janata and Cajthaml, 2018).

\subsection{Vltava Course}

The Vltava River is the longest river in the Czech Republic with a length of $430.2 \mathrm{~km}$. It originates in the Šumava Mountains, near the village Černý Kříž, as the confluence of the Teplá Vltava River and the Studená Vltava River. It flows through Český Krumlov, České Budějovice and Prague, and flows from the left to the Labe River in Mělník. The Vltava River has long been used as an important waterway. Therefore, many weirs with passes, waterfront walls and other constructions were built since the $18^{\text {th }}$ century. The set of large hydrotechnic works on the Vltava River is called the Vltava Cascade. There are 9 dams in the cascade. The first of them - Vrané nad Vltavou - was built in the 1930 s, the last of them - Hněvkovice - was completed in 1990s. The Vltava Cascade includes a dam reserving the largest volume of water from the Czech reservoirs (Orlík) as well as the largest dam in the surface area (Lipno). Hydroelectric power plants in cascade dams produce an electrical output of up to $750 \mathrm{MW}$.

\subsection{Old Maps}

There are diverse archival materials through which we can get a lot of information about the original Vltava River. The basic source of data for the creation of the model of the historical Vltava valley is the old maps, which precede the construction of dams by their date of origin. With regard to the extent of the territory - in the project the Vltava will be processed from the source to the confluence with the Berounka River - it is necessary to choose a map series that covers the whole area of interest and contains the elevation component necessary for the creation of the 3D model. These conditions are met by a large scale state map work State Map 1:5000derived (SMO-5) in the first edition.

SMO-5 has been released since 1950. Initially, it was intended to serve as a provisional and as a replacement for the State Map of Czechoslovakia 1:5000, whose creation was not fast enough. SMO-5, on the other hand, was created exclusively by reworking existing documents, not by direct measurements. It thus fulfilled the requirement for the entire territory to be covered as soon as possible. The planimetry component of the map was in the first edition derived from cadastral maps and the altimetry component from topographic maps created in the S-1952 system; in some cases also from the topographic sections of the 3rd Military Mapping of the Habsburg Empire. The altimetry, which will be the subject of subsequent processing, was represented by brown lines and consisted of contour lines, elevation points, and, where appropriate, technical or topographical hatching and description. Altitudes are given in the Baltic altitude system after alignment. Each map sheet displayed an area of $2.5 \times 2 \mathrm{~km}$. SMO-5 has been released several 
times, and until 1990 it was only available for the state purposes. Currently, the first issue of the SMO-5 map can be purchased as an archive material at the Geoportal of the Czech Office for Surveying, Mapping and Cadastre. The data is available in digital form (JPEG files). Our area of interest consists of 334 map sheets of the first edition of SMO-5.

The altimetry on the processed map sheets was, in most cases, derived from topographic maps of the scales $1: 10,000$ and $1: 25,000$ according to the metadata of the map. The following table shows the contour intervals that appear on the selected map sheets, including their share in the total area of the territory.

\begin{tabular}{|r|r|}
\hline $\begin{array}{r}\text { Contour } \\
\text { interval }\end{array}$ & Share \\
\hline $1 \mathrm{~m}$ & $2.7 \%$ \\
$2 \mathrm{~m}$ & $8.2 \%$ \\
$5 \mathrm{~m}$ & $5.7 \%$ \\
$10 \mathrm{~m}$ & $59.8 \%$ \\
$20 \mathrm{~m}$ & $23.6 \%$ \\
\hline
\end{tabular}

Table 1. SMO-5 contour intervals in the area of interest

\subsection{Longitudinal profile}

Another archival material, namely a longitudinal profile of the Vltava River from 1940, which accurately describes the height ratios of the river and its banks, is utilized in our case. The profile was created by the State Hydrologic Institute in Prague during World War II. It covers the most important part of the Vltava River for the industry and transportation - from České Budějovice to Prague. Lengths are depicted in 1:10,000 while heights are depicted in 1:200. The covered area is displayed very precisely, with all height marks (not even on the river banks), flood marks, hydrotechnic objects, confluences with other rivers and streams etc. The profile contains information about river banks heights and the most damaging historic floods as well.

\subsection{Modelling of 3D valley}

To create a 3D model of the valley, it is crucial to obtain the vector spatial data, in our case contours, elevation points and hatching. The automatic vectorization of contours has been solved in a number of publications, but this is a complex problem. The source raster data must first be edited and cleaned. This may then be followed by automatic or semi-automatic vectorization. Finally, the appropriate attributes need to be assigned to the vector elements, data should be consolidated and checked. (Pacina and Havlicek, 2015) describe an example of creating 3D model from digitized contours.

\section{Methodology}

\subsection{Data preparation}

In the preparation phase, it is necessary to convert the elevation content of the map sheets into a vector form. Vectorization can generally be performed manually, semi-automatically or automatically. The appropriate method should be chosen with regard to the type, quantity and quality of the input data. In this case, due to the total volume of vectorized data, the method of automatic vectorization was chosen with subsequent manual addition of attributes and eventual manual editing. Automatic vectorization was performed using ArcScan (ArcGIS extension).

Before starting the vectorization it was necessary to edit the raster files. Such editing can be divided into three phases: raster placement in the reference coordinate system, raster adjustments needed for proper working in ArcScan, and raster editing in ArcScan (raster preprocessing). First, there was need to place scanned map sheets in the reference coordinate system; in our case they were georeferenced into the Czech national coordinate system S-JTSK. Transformation of all map sheets was performed using the ArcGIS and projective transformation. This kind of transformation is often used within georeferencing of old maps. To solve the transformation parameters, it is essential to determine at least 4 identical points - in our case 4 corners of map.

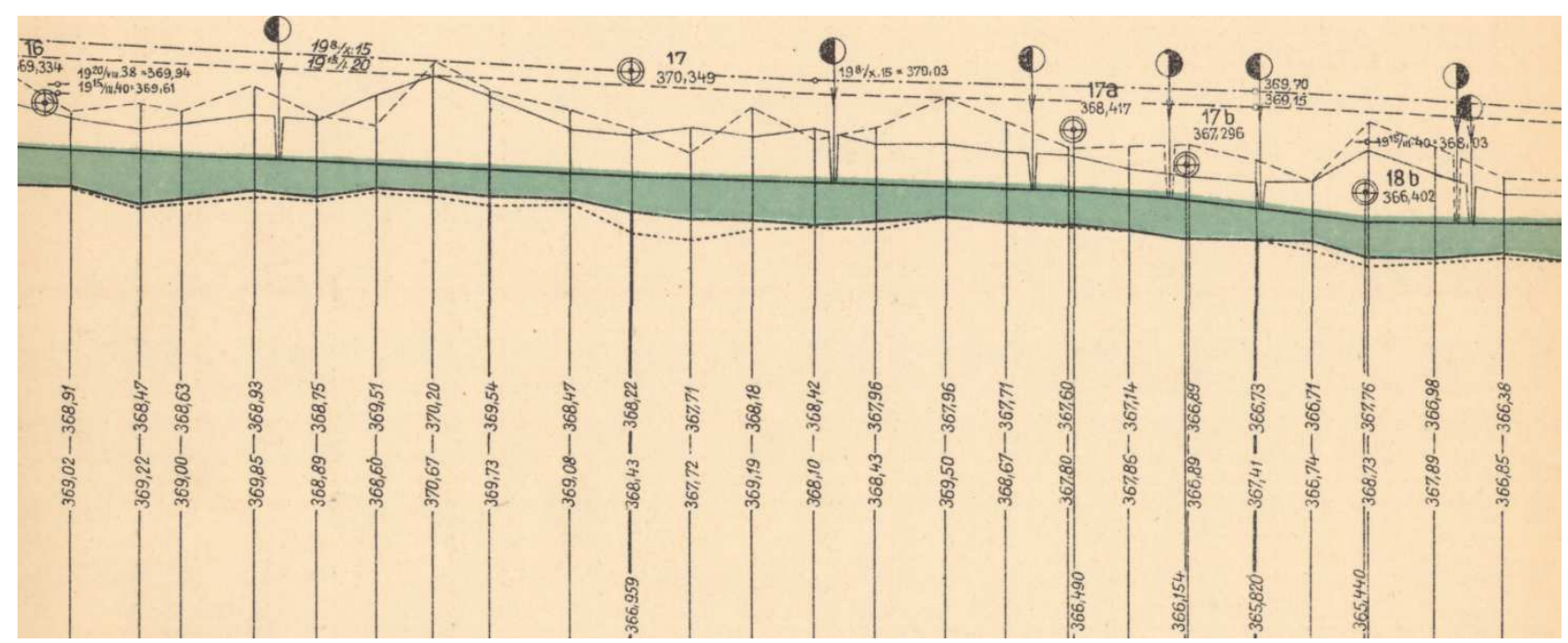

Figure 1. Part of the longitudinal profile from 1940 
The georeferenced map sheets were then merged into a seamless mosaic, which can be used as a texture for further visualization of the valley. Map sheets were processed individually during the process of vectorization.

ArcScan extension requires a binary raster file input and therefore it was necessary to reduce the colour depth of the data. The shades of brown form one colour of the resulting raster (foreground), the other colours form a second colour (background). On some map sheets we unfortunately separated not only altimetric features but some parts of the planimetric part as well (the gray colour was due to JPEG compression approaching brown). Several morphological operations were used to reduce such errors.

\subsection{Vectorization}

With standard automatic vectorization procedure, we created vector data of contour lines. All ArcScan vector files were then manually edited using ArcGIS standard editing tools. Altitude according to raster background had to be added to the attribute table of each contour line. Simultaneously, vectorization of elevation points from all map sheets was made to the point shapefile. Errors in the description of contour lines in underlying maps were revealed in several places when editing vectorized data. After editing all map sheets, the shapefiles were merged into the united geodatabase.

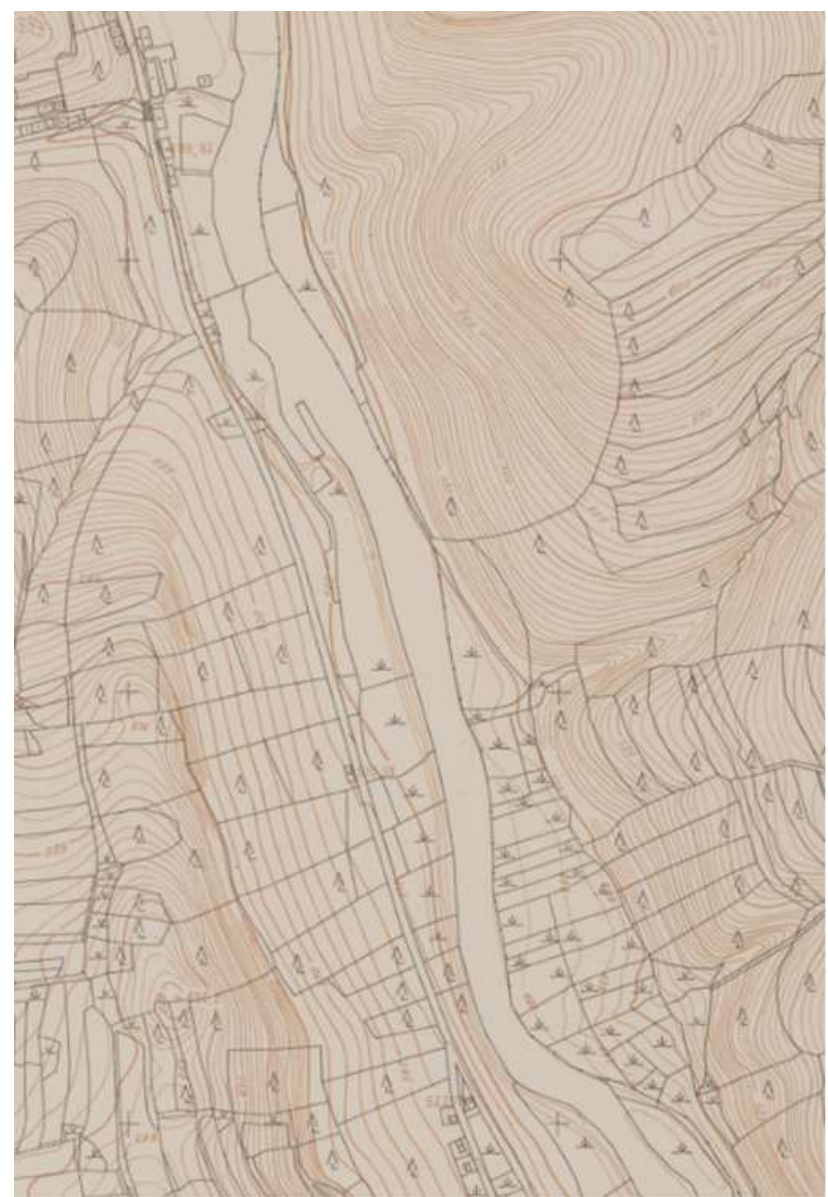

Figure 2. SMO-5 and final vectorized data
Subsequently, the linkage of lines on the edges of the map sheets had to be solved, where the contour drawing was not continuous with respect to the previous processing on the individual map sheets. The ETGeoWizards Clean Contour Gaps tool was used to link contour lines between map sheets. See final results on the Figure 2.

In the case of longitudinal profile it was necessary to vectorize the $2 \mathrm{D}$ polygon of the river from the SMO-5 and to add altitudes from the longitudinal profile to the vertices of this polygon. The spot-height of the river level appears in the profile approx. by $1 \mathrm{~km}$, and thus heights of vertices are interpolated by Python script. The result of this phase is the creation of the $3 \mathrm{D}$ river level polygon that respects the longitudinal profile and the planimetry of the Vltava River.

\subsection{D model creation}

After creating vector lines and point elevation data, it was our aim to create a 3D model of the historic Vltava valley. This model can be in vector form as TIN (triangulated irregular network), but more often it is used in GIS as a DEM (digital elevation model) in the form of a raster grid. There are a number of publications dealing with creating such model. Most GIS software includes tools for creating DEM. Algorithms work either at the

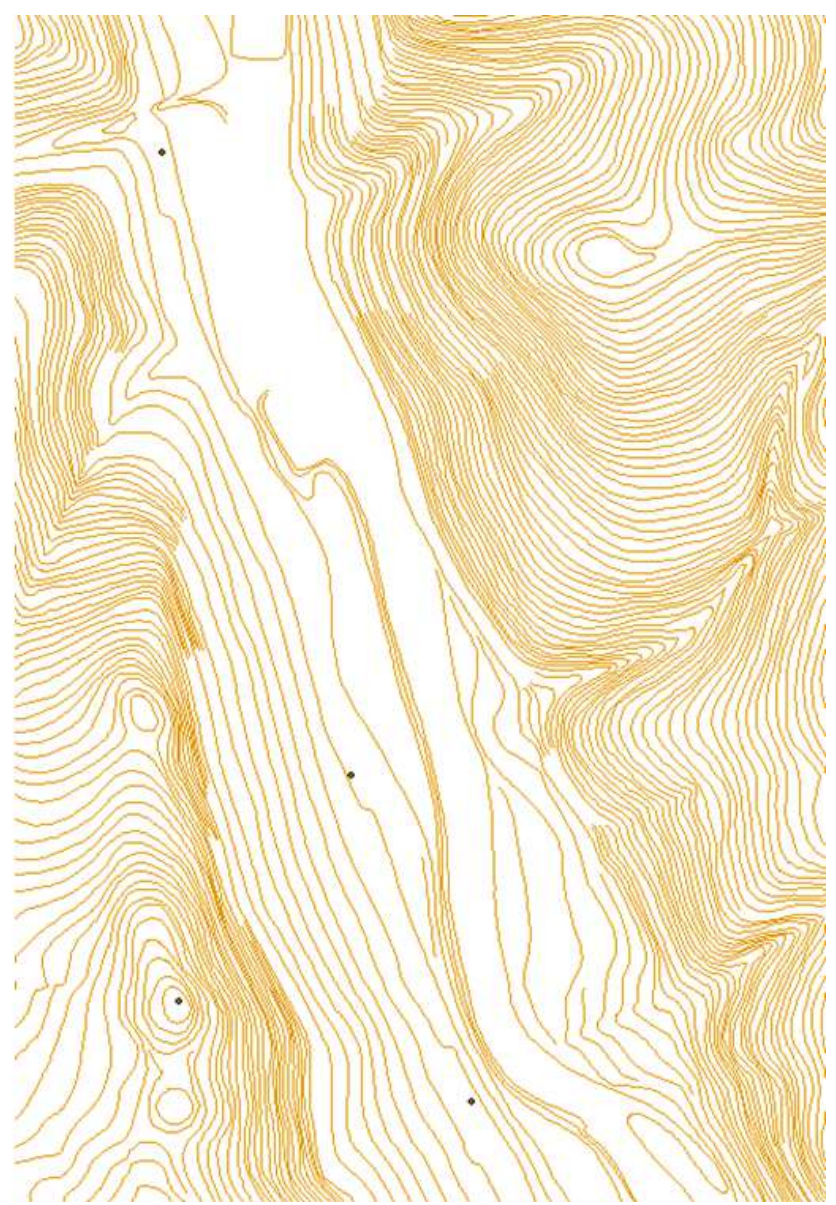




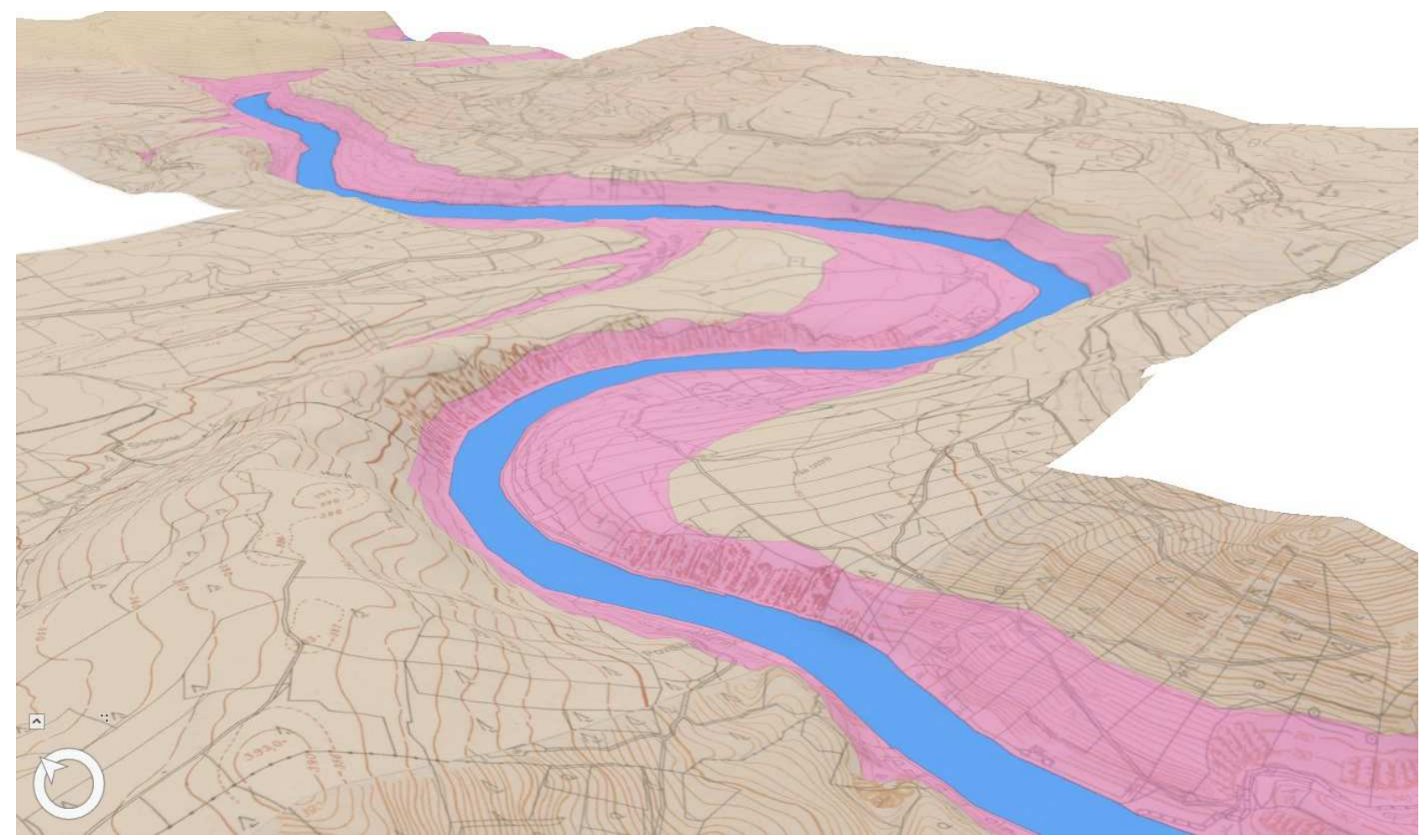

Figure 3. 3D model of historic Vltavy valley

point level (lines are replaced by points at vertices) and subsequent interpolation of point data into a grid, or are more complex algorithms working with hydrological correct models, distinguishing individual types of input data. Interpolation methods use natural neighbours, IDW (inverse distance weighted) or more complex mathematical surfaces, such as splines. See their comparison in (Arun, 2013). In our case, we have stayed with ArcGIS for Desktop, which includes Topo To Raster routine. Topo To Raster tool is an interpolation method specifically designed for the creation of hydrological correct digital elevation models (DEMs). It is based on the ANUDEM programme developed by Michael Hutchinson. Applications of DEMs to environmental modelling are discussed in (Hutchinson and Gallant, 2000) and (Hutchinson, 2008). Input data for our calculation came not only from the contours and elevation points, but also from the $3 \mathrm{D}$ river level polygon created with respect to the longitudinal profile. After testing the input data and the output parameters settings, DEM was created with a 2-meters spatial resolution. The resulting DEM can be visualized as a 3D textured surface. In our case, it is possible to use map SMO-5, or black and white orthophoto from the 1950s (see Figure 3).

\section{Conclusion}

The resulting vector data was obtained by combining automatic and manual vectorization. The automatic vectorization method was chosen with respect to the amount of vectorized data. However, it required a fairly extensive adjustment of the input raster data. Automatic vectorization was performed over modified raster data in the ArcScan extension of ArcGIS. Optimized vectorization parameters can be used for most map sheets. The degree to which manual vectorization was subsequently used was different depending on the quality of the output vector file from ArcScan. Here, the properties of the raster file and the quality of its edits that preceded the vectorization played a key role. It was always necessary to add the altitude attribute to the individual contours at least, or to edit the contour drawing manually. In areas of dense contour interval and steep terrain, where the distances of each contour in the map were minimal, manual vectorization was necessary.

\section{Acknowledgements}

This work was supported by the Ministry of Culture of the Czech Republic by the NAKI programme "Vltava transformation of historical landscape as a result of floods, dams' creation and land-use changes along with cultural and social activities in the river neighbourhood" no. DG18P02OVV037.

\section{References}

Arun, P.V. (2013). A comparative analysis of different DEM interpolation methods, The Egyptian Journal of Remote Sensing and Space Science 16(2): 133-139.

Hutchinson, M.F. (2008). Adding the Z-dimension, Handbook of Geographic Information Science, Oxford: Blackwell.

Hutchinson, M.F. and Gallant, J.C. (2010). A Digital elevation models and representation of terrain shape, Terrain Analysis. New York: Wiley. 
Janata, T. and Cajthaml, J. (2018). Vltava transformation of historical landscape along with cultural and socioeconomic activities in the river neighbourhood, Proceedings 13th ICA Conference Digital Approaches to Cartographic Heritage, April 1820, 2018, Madrid.

Pacina, J. and Havlicek, J. (2015). A vanished settlement in the Ore Mountains - the creation of 3D models, Advances and Trends in Engineering Sciences and Technologies, International Conference on Engineering Sciences and Technologies (ESaT), May 27-29, 2015, Kosice. 